\title{
Inhibition of FOXM1 by thiostrepton sensitizes medulloblastoma to the effects of chemotherapy
}

\author{
JIAPING LIN*, YIPENG ZHENG* ${ }^{*}$ KUN CHEN, ZHENGSONG HUANG, \\ XINJIAN WU and NU ZHANG \\ Department of Neurosurgery, The First Affiliated Hospital of Sun Yat-Sen University, \\ Guangzhou, Guangdong 510080, P.R. China
}

Received June 13, 2013; Accepted July 22, 2013

DOI: $10.3892 / o r .2013 .2654$

\begin{abstract}
Medulloblastoma (MB) is the most common malignant brain tumor in children and is highly invasive and metastatic. Despite recent advances, most MB patients suffer significant therapy-related morbidity, and the survival rate for patients with metastatic MB remains unsatisfactory. Altered expression of FOXM1 has been detected in many types of cancers, and the inhibition of FOXM1 has been studied as a cancer therapy. In the present study, we evaluated the impact of the inhibition of FOXM1 by thiostrepton in Daoy MB cells. Cells were treated with different concentrations of thiostrepton alone or in combination with cisplatin. Cell viability was measured with CCK-8 assays, and cell cycle distribution and apoptosis were assessed by flow cytometric analysis. Changes in protein expression were examined by western blotting. RNAi experiments were performed using siRNA oligonucleotides. The invasion and migration studies were performed using $8-\mu \mathrm{m}$ Transwell plates. Inhibition of FOXM1 by thiostrepton significantly decreased MB cell proliferation. Cell arrest at the G2/M phase and apoptosis were significantly increased in MB cell lines that were treated with thiostrepton or transfected with siRNA. Thiostrepton decreased the $\mathrm{IC}_{50}$ value of cisplatin for MB treatment by enhancing cisplatininduced apoptosis. Thiostrepton also decreased cell invasion and migration, which are crucial steps for tumor progression. Our data suggest that targeting FOXM1 with small-molecule inhibitors results in potent antitumor activity and chemosensitizing effects in human medulloblastoma cells.
\end{abstract}

Correspondence to: Dr Xinjian Wu or Dr Nu Zhang, Department of Neurosurgery, The First Affiliated Hospital of Sun Yat-Sen University, No. 58, Zhongshan 2 Road, Guangzhou, Guangdong 510080, P.R. China

E-mail:wuxinjian@msn.com

E-mail: zhangxiaonu@gmail.com

*Contributed equally

Key words: thiostreptin, FOXM1, medulloblastoma, chemotherapy

\section{Introduction}

Medulloblastoma (MB) is the most common pediatric malignant brain tumor, and satisfactory treatment results are notoriously difficult to achieve. MB has a high propensity to metastasize, as $\sim 30 \%$ of patients have evidence of leptomeningeal dissemination at initial diagnosis (1). Despite recent advances in surgery, radiotherapy and chemotherapy, only 60 to $75 \%$ of affected children are cured, and the majority of them suffer from considerable long-term morbidity after aggressive multimodal therapy (2). Attempts to further improve outcomes and decrease the morbidity associated with MB have been restricted by the conventional cytotoxic approaches used and the infiltrative nature of the disease (2-4). Therefore, increased understanding of the mechanisms underlying MB is necessary to develop novel therapeutic approaches.

The oncogenic transcription factor FOXM1 is known to stimulate proliferation by promoting cell cycle transition and is involved in the proper execution of mitosis $(5,6)$. Elevated expression of FOXM1 has been detected in a wide range of human tumors and has been implicated in cellular transformation, tumor initiation and progression (7). Accumulating evidence demonstrates that increased expression of FOXM1 is associated with poor prognosis in various types of cancers (8-11), including MB (12). Furthermore, previous studies have shown that FOXM1 mediates resistance to a diverse spectrum of anticancer drugs in breast cancer (13-15). A recent study confirmed that suppression of FOXM1 enhanced the chemosensitivity of various types of cancer cells to the DNA-damaging reagent doxorubicin (16). Therefore, the inhibition of FOXM1 activity has emerged as an attractive goal for cancer therapy. However, since FOXM1 displays a proliferation-specific expression pattern and is essential for embryonic development (5), the impact of inhibiting FOXM1 activity in non-malignant cells must be considered.

Thiostrepton, a natural product originally isolated from Streptomyces azureus, has captured a great deal of attention because of its potent anticancer activity as a FOXM1 inhibitor (17-19). The mechanism by which thiostrepton affects FOXM1 remains unknown. Hegde et al (18) reported that thiostrepton interacts directly with FOXM1 protein to inhibit the transcriptional activity of FOXM1, whereas Bhat et al (19) suggested that thiostrepton functions as a proteasomal 
inhibitor. However, in a wide variety of tumor cell types, thiostrepton-induced apoptosis is dependent on the expression of FOXM1 $(10,17,20)$. Importantly, thiostrepton appears to exert minimal toxicity against non-malignant cells $(17,21)$. Thiostrepton has shown anticancer activity in rodent xenograft models without observable toxicity $(10,22,23)$. Collectively, these data suggest that thiostrepton is an ideal treatment for $\mathrm{MB}$, particularly in children. In this study, we examined the antitumor effects of thiostrepton in Daoy MB cells. More importantly, we assessed the ability of thiostrepton to sensitize MB cell lines to cisplatin, which is commonly used for the treatment of MB.

\section{Materials and methods}

Chemicals and reagents. Thiostrepton purchased from Tocris Cookson Inc. (Ellisville, MO, USA) was freshly dissolved in dimethyl sulfoxide (DMSO) to make a $10 \mathrm{mmol} / \mathrm{l}$ stock solution. Cisplatin (Qilu Pharmaceutical Co., Ltd., Shandong, China) was dissolved at a stock concentration of $2 \mathrm{mmol} / \mathrm{l}$ and divided into aliquots. An antibody specific for FOXM1 was obtained from Santa Cruz Biotechnology Inc. (Santa Cruz, CA, USA) Antibodies specific for Bcl-2, Bax, caspase-3, PARA and $\beta$-actin were obtained from Cell Signaling Technology, Inc.

Cell culture. The Daoy human MB cell line was obtained from ATCC (Manassas, VA, USA) and maintained in Dulbecco's modified Eagle's medium (DMEM) supplemented with $10 \%$ fetal bovine serum (FBS) (both from Gibco-BRL) and incubated at $37^{\circ} \mathrm{C}$ in a humidified incubator in the presence of $5 \%$ $\mathrm{CO}_{2}$.

siRNA transfection. RNA interference was performed by transfecting Daoy cells with 21-nucleotide RNA duplexes. FOXM1 siRNA and mock siRNA were synthesized by Shanghai GenePharma Co., Ltd. (Shanghai, China). The siRNA-NC did not target any known mammalian gene and was synthesized by Shanghai GenePharma Co., Ltd. siRNA transfection was carried out with Lipofectamine 2000 (Invitrogen Life Technologies, Carlsbad, CA, USA), according to the procedure recommended by the manufacturer. Six hours after transfection with siRNA NC at various concentrations, the cells were analyzed on a FACSCalibur flow cytometer equipped with Cell Quest software (Becton-Dickinson, San Jose, CA, USA).

Western blot analysis. The cells were collected and lysed in lysis buffer on ice. The cell lysates were centrifuged at 10,000 $\mathrm{x}$ g for $10 \mathrm{~min}$ at $4^{\circ} \mathrm{C}$, and the protein content in the supernatants was determined using a BCA protein assay kit (Pierce Biotechnology, Inc., USA). Equal amounts of protein lysate were electrophoretically separated on $10 \%$ sodium dodecyl sulfate-polyacrylamide gels and transferred to polyvinylidene difluoride (PVDF) membranes (Millipore, USA). The membranes were blocked with $1 \%$ bovine serum albumin (BSA) for $2 \mathrm{~h}$ at room temperature and then incubated with anti-FOXM1, anti-Bcl-2, anti-Bax, anti-caspase 3 , anti-PARA or anti- $\beta$-actin primary antibody overnight at $4^{\circ} \mathrm{C}$. Horseradish peroxidase (HRP)-conjugated secondary antibody was added for $2 \mathrm{~h}$ at room temperature. Detection was performed by enhanced chemiluminescence (ECL; Pierce Biotechnology, Inc.).
Cell proliferation analysis. Cell proliferation assays were performed using the CCK-8 kit (Cell Counting Kit-8; Dojindo Laboratories), according to the manufacturer's instructions. In brief, Daoy cells were seeded in a 96-well plate at a density of $5 \times 10^{4}$ cells $/ \mathrm{ml}$. The following day, the medium was replaced with DMEM containing 10\% FBS with or without agents. The cells were incubated for 24,48 or $72 \mathrm{~h}$, and CCK- 8 was used according to the manufacturer's instructions. Extinction was measured at $450 \mathrm{nM}$, and the reference extinction was subtracted. Each experiment was performed in triplicate and repeated 3 times.

Cell cycle analysis. Daoy cells were seeded in 6-well plates in DMEM containing 10\% FBS. The following day, the medium was replaced with DMEM containing 10\% FBS with or without agents. The cells were detached after $24 \mathrm{~h}$ and fixed with $500 \mu \mathrm{l}$ of $70 \%$ ethanol at $-20^{\circ} \mathrm{C}$ for $2 \mathrm{~h}$. Subsequently, the cells were washed twice with PBS and then stained with propidium iodide (PI) $(50 \mu \mathrm{g} / \mathrm{ml}$ propidium iodide and $100 \mu \mathrm{g} / \mathrm{ml}$ RNase A in PBS) at $37^{\circ} \mathrm{C}$ for $30 \mathrm{~min}$. Cell cycle analysis was performed on a FACScan flow cytometer (Becton-Dickinson).

Apoptosis analysis. Daoy cells were seeded in 6-well plates in DMEM containing 10\% FBS. The following day, the medium was replaced with DMEM containing 10\% FBS without or with agents. The cells were collected after $24 \mathrm{~h}$, and cell apoptosis was detected by Annexin V-FITC/PI staining. The experiments were performed in triplicate for each sample, and analyses were performed using a FACScan flow cytometer in accordance with the manufacturer's guidelines.

Colony formation assays. Daoy cells growing in log phase were seeded at a density of 1,000 cells/well in a 6-well plate in complete growth medium containing $10 \%$ FBS. The cells were allowed to adhere for $24 \mathrm{~h}$, and the medium was replaced with fresh complete growth medium containing the indicated concentrations of thiostrepton. The cells were cultured at $37^{\circ} \mathrm{C}$ for 10 days with medium changes every third or fourth day. Colony formation was detected by crystal violet staining.

Cell invasion and migration assay. Daoy cells were treated with the indicated concentrations of thiostrepton for $48 \mathrm{~h}$, and equal numbers of cells were suspended in serum-free medium and seeded into either uncoated Transwell inserts (for migration assays) or growth factor-reduced Matrigel-coated Transwell inserts (for invasion assays) (BD Biosciences, Bedford, MA, USA). The bottom wells were filled with complete medium, and after $12 \mathrm{~h}$, the cells were stained with crystal violet and photographed under a fluorescence microscope. The number of cells that penetrated the membrane was determined by counting the mean cell number of 5 randomly selected highpower fields.

Statistical analysis. All data were analyzed using GraphPad Prism version 5 (GraphPad Software Inc., La Jolla, CA, USA). $t$-tests were used for pairwise comparisons. The synergistic or antagonistic effects of 2 drugs were evaluated according to the formula $[\mathrm{Q}=\mathrm{Ea}+\mathrm{b} / \mathrm{Ea}+\mathrm{Eb}-\mathrm{Ea} \times \mathrm{Eb}]$. In this equation, $\mathrm{Ea}+\mathrm{b}$ represents the inhibition rate of the combined drug therapy on tumor cell proliferation, and Ea 

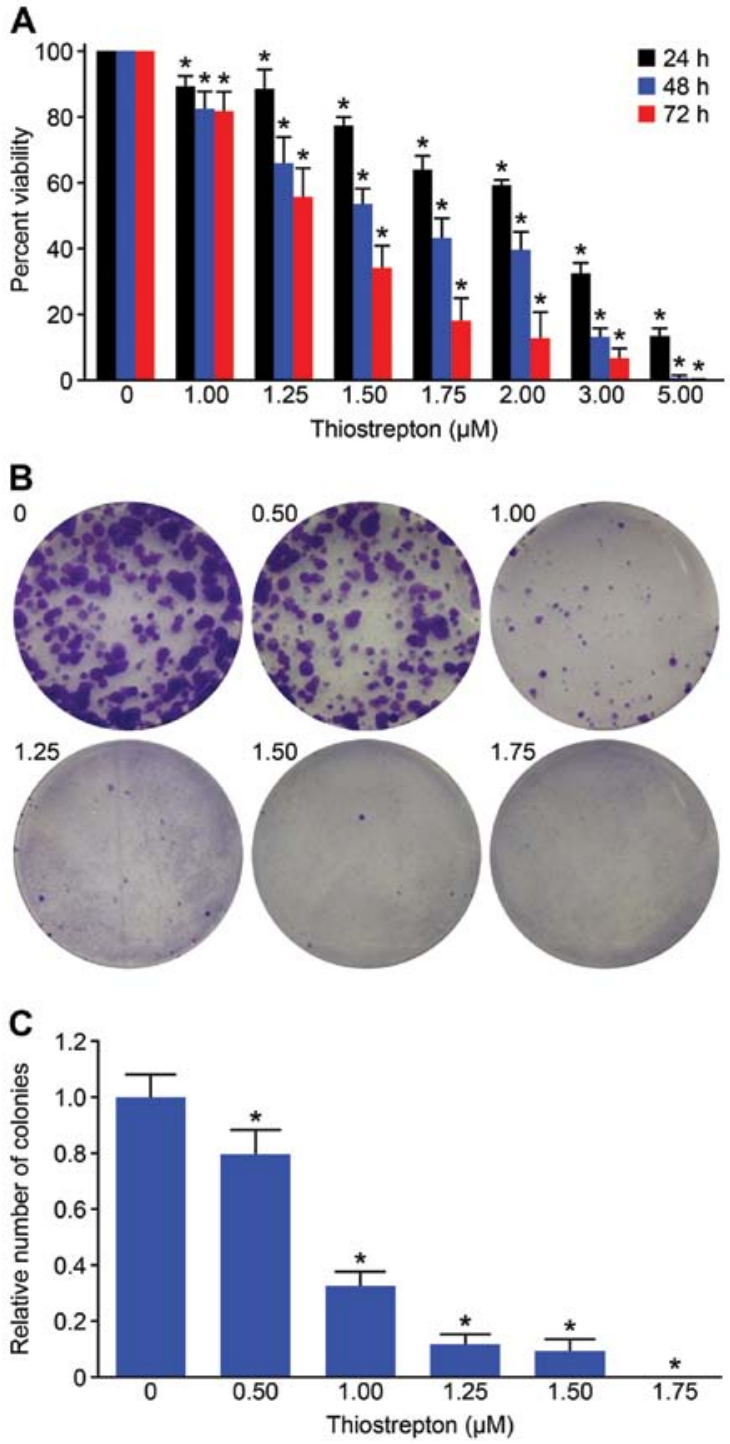

Figure 1. Thiostrepton inhibits cell proliferation and decreases the colony formation capacity of Daoy cells. (A) Daoy cells were incubated with various concentrations of thiostrepton for 24,48 or $72 \mathrm{~h}$. Cell viability was assessed by CCK- 8 assay. (B) Following treatment with the indicated concentrations of thiostrepton, the clonogenicity of Daoy cells was determined. (C) The graph displays the means \pm SD of 3 independent clonogenicity formation experiments. ${ }^{*} \mathrm{P}<0.05$, statistically significant (Student's t-test).

and $\mathrm{Eb}$ represent the inhibition rates of drug $\mathrm{A}$ and drug $\mathrm{B}$ individually. Q-values ranging from 0.85 to 1.15 indicate that the effects of the 2 drugs are simply additive, Q-values $>1.15$ indicate a synergistic effect, and $\mathrm{Q}$-values $<0.85$ indicate an antagonistic effect for the combined drug therapy.

\section{Results}

Thiostrepton inhibits cell proliferation and decreases the colony formation capacity of Daoy cells. We first investigated the effect of thiostrepton on Daoy cell proliferation. Daoy cells were treated with various concentrations of thiostrepton $(1-5 \mu \mathrm{M})$ for 24,48 or $72 \mathrm{~h}$, and cell proliferation was measured by performing CCK-8 assays. Our results demonstrated that Daoy cells were sensitive to thiostrepton, and thiostrepton inhibited cell growth in a time- and dose-dependent manner $(\mathrm{P}<0.05)$ (Fig. 1A). The $\mathrm{IC}_{50}$ values of Daoy cells to thio-
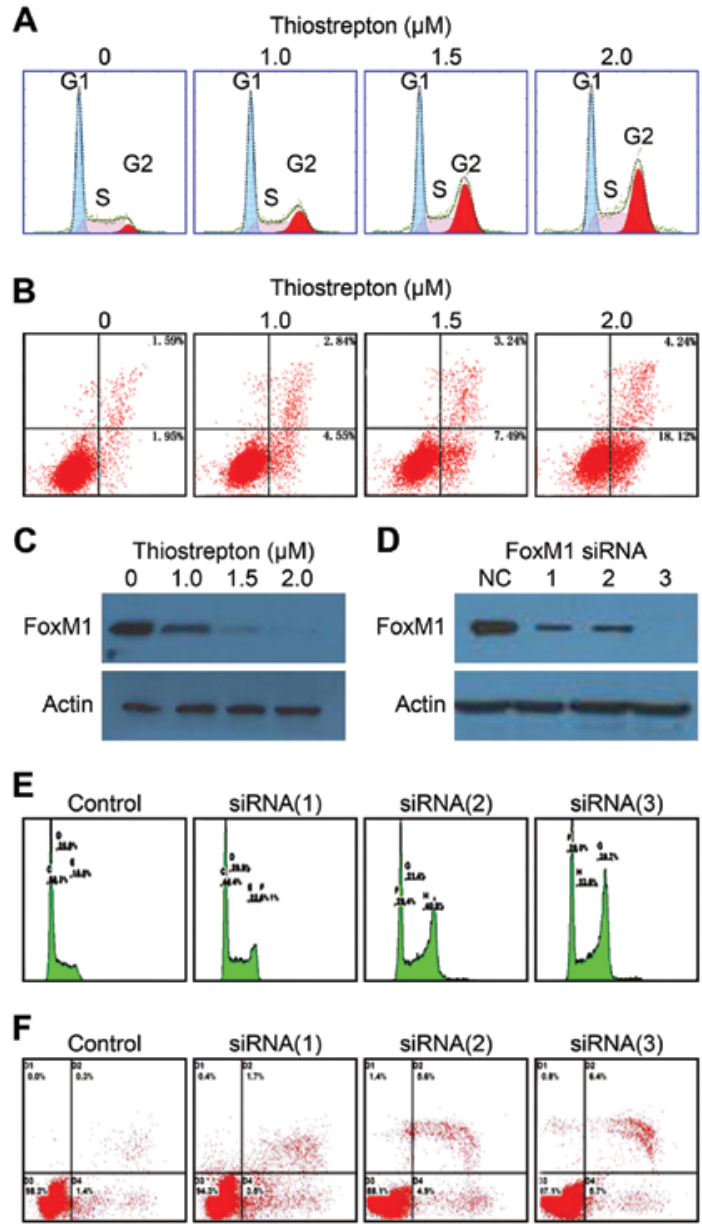

Figure 2. Inhibition of FOXM1 by thiostrepton induces cell cycle arrest and leads to apoptosis of Daoy cells. (A-C) Thiostrepton inhibits FOXM1 expression and induces G2-M arrest and apoptosis in a dose-dependent manner. (D) Daoy cells were transfected with either negative control (NC) siRNA or 3 FOXM1-specific siRNAs, which targeted different sites of the FOXM1 gene. FOXM1 protein levels were determined by western blotting. All 3 FOXM1 siRNAs induced (E) G2-M arrest and (F) apoptosis in Daoy cells.

Table I. $\mathrm{IC}_{50}$ values for thiostrepton in Daoy cells were calculated at 24,48 or $72 \mathrm{~h}$.

\begin{tabular}{lccc}
\hline & \multicolumn{3}{c}{ Treatment time } \\
\cline { 2 - 4 } & $24 \mathrm{~h}$ & $48 \mathrm{~h}$ & $72 \mathrm{~h}$ \\
\hline $\mathrm{IC}_{50}(\mu \mathrm{M})$ for thiostrepton & 2.14 & 1.69 & 1.39 \\
\hline
\end{tabular}

strepton after 24,48 or $72 \mathrm{~h}$ of treatment are shown in Table I. To evaluate the long-term impact of thiostrepton on MB cells, we performed colony formation assays. We found that Daoy cells had a robust ability to form colonies, and colony formation was significantly inhibited by thiostrepton. Treatment with $1.75 \mu \mathrm{M}$ thiostrepton completely abolished the colony formation ability of Daoy cells (Fig. 1B and C).

Thiostrepton-mediated inhibition of FOXM1 induces cell cycle arrest and leads to apoptosis of Daoy cells. To further characterize the mechanism underlying the antitumor activity 
Table II. Analysis was employed to characterize the interactions between the drugs.

\begin{tabular}{cccccccrrr}
\hline & \multicolumn{7}{c}{ Cisplatin $(\mu \mathrm{M})+$ Thio $(1 \mu \mathrm{M})^{\mathrm{a}}$} \\
\cline { 2 - 8 } & 1 & 2 & 3 & 4 & 5 & 10 & 20 & 40 \\
\hline Q-value & 1.35 & 1.31 & 1.78 & 1.66 & 1.29 & 1.04 & 1.03 & 1 \\
\hline
\end{tabular}

$\mathrm{Q}>1.15$ corresponds to a synergistic interaction; ${ }^{\mathrm{a}}$ For all the various concentrations of cisplatin, $1 \mu \mathrm{m}$ thiostrepton was used.

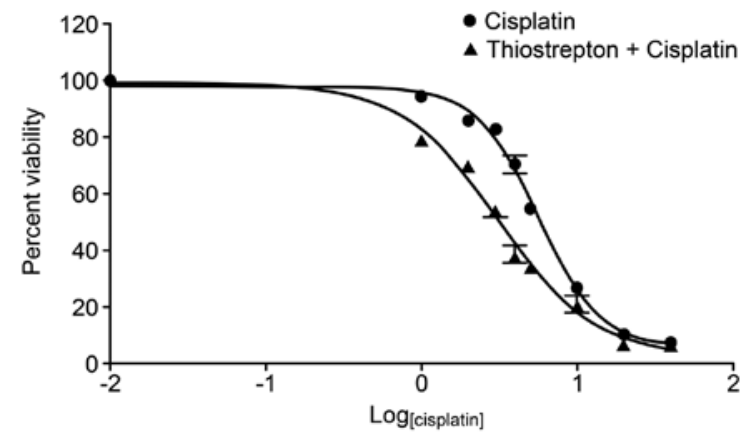

Figure 3. Thiostrepton acts synergistically with cisplatin to inhibit the viability of Daoy cells. Daoy cells were co-treated with thiostrepton and cisplatin, resulting in a significant decrease in the $\mathrm{IC}_{50}$ value of cisplatin.

of thiostrepton on Daoy cells, we investigated the expression of FOXM1 following thiostrepton treatment and examined whether thiostrepton affects cell cycle distribution and apoptosis. The results of these experiments showed that thiostrepton inhibited FOXM1 expression in a dose-dependent manner and induced a gradual dose-dependent $\mathrm{G} 2 / \mathrm{M}$ arrest and apoptosis in Daoy cells (Fig. 2A-C). To validate the target specificity of thiostrepton, we downregulated FOXM1 expression using RNAi. Three different FOXM1 siRNA oligonucleotides, but not the negative control (NC) oligonucleotides, efficiently knocked down FOXM1 protein expression (Fig. 2D) and similarly resulted in a significant $\mathrm{G} 2 / \mathrm{M}$ cell cycle phases arrest and apoptosis in Daoy cells (Fig. 2E and F). These results indicate that thiostrepton induces G2/M cell cycle phase arrest and leads to apoptosis in Daoy cells by targeting FOXM1.

Thiostrepton enhances the anti-proliferative effects of cisplatin on Daoy cells. The upregulation of FOXM1 has recently been reported to be closely related to chemotherapy resistance, and the inhibition of FOXM1 has been shown to increase the sensitivity of tumor cells to chemotherapeutic agents $(13,15,24-26)$. We next aimed to ascertain whether combining FOXM1 inhibition with standard chemotherapeutic agents would enhance the effect. Daoy cells were cultured in the presence of cisplatin $(0-40 \mu \mathrm{m} / \mathrm{l})$ with or without thiostrepton $(1 \mu \mathrm{m} / \mathrm{l})$ for $24 \mathrm{~h}$. Cytotoxicity was assessed using CCK-8 proliferation assays. The results demonstrated that combined treatment reduced the $\mathrm{IC}_{50}$ value of cisplatin in Daoy cells from 5.622 to $3.116 \mu \mathrm{mol} / 1$ (Fig. 3). Thiostrepton and cisplatin were shown to synergistically inhibit cell growth $(\mathrm{Q}>1.15)$ when the cells were treated with suboptimal concentrations of cisplatin (Table II).
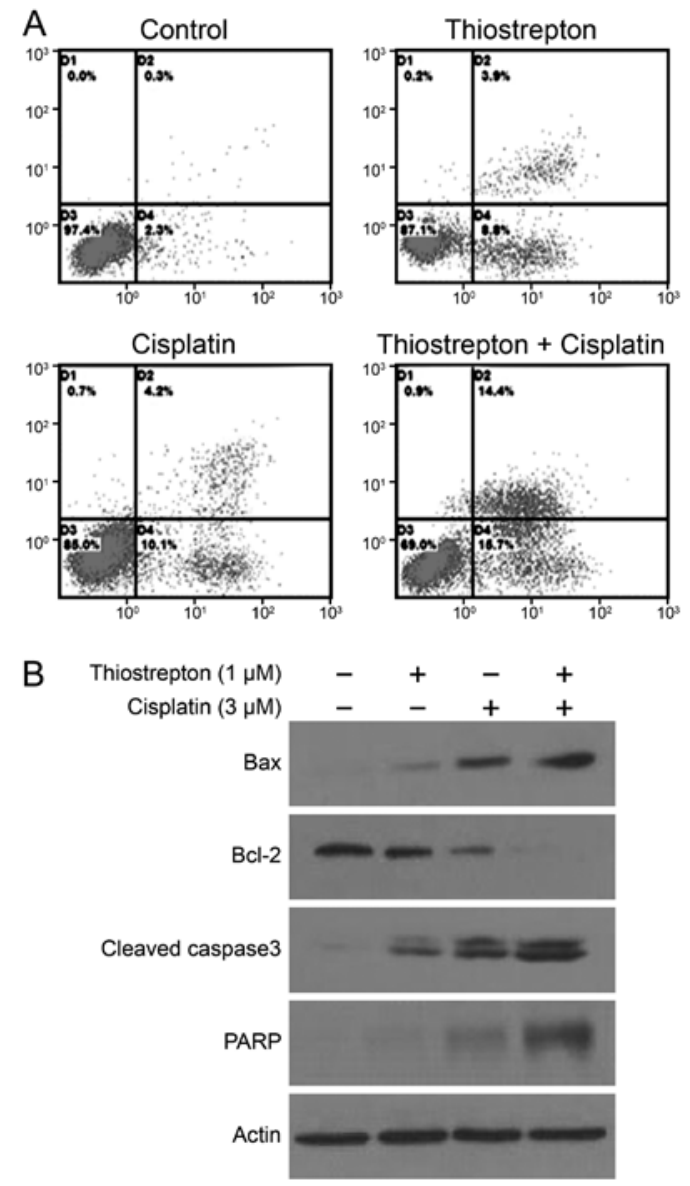

Figure 4. Thiostrepton enhances the anti-proliferative effects of cisplatin on Daoy cells. (A) Treatment with a combination of $1 \mu \mathrm{M}$ thiostrepton and $3 \mu \mathrm{M}$ cisplatin significantly increased Daoy cell apoptosis when compared with either drug alone $(\mathrm{P}<0.01)$. (B) The expression of apoptosis-related proteins was detected by western blotting.

Thiostrepton enhances cisplatin-induced caspase-mediated apoptosis in Daoy cells. To characterize thiostrepton-mediated apoptotic enhancement, Daoy cells were treated with a low concentration of either thiostrepton $(1 \mu \mathrm{m} / \mathrm{l})$ or cisplatin $(3 \mu \mathrm{m} / \mathrm{l})$ or treated with both drugs for $24 \mathrm{~h}$. Flow cytometric analysis showed that thiostrepton increased cisplatin-induced apoptosis from $14.3 \pm 2.46$ to $30.2 \pm 3.16 \%$ ( $\mathrm{P}<0.01$ ) (Fig. 4A). To gain a better understanding of the mechanism leading to cell death, we measured the combined effect of thiostrepton and cisplatin on the expression of Bcl-2, Bax, caspase-3 and PARP proteins. As shown in Fig. 4B, the decreases in Bcl-2 expression were significantly greater in samples treated with both thiostrepton $(1 \mu \mathrm{M})$ and cisplatin $(3 \mu \mathrm{M})$ than those in 

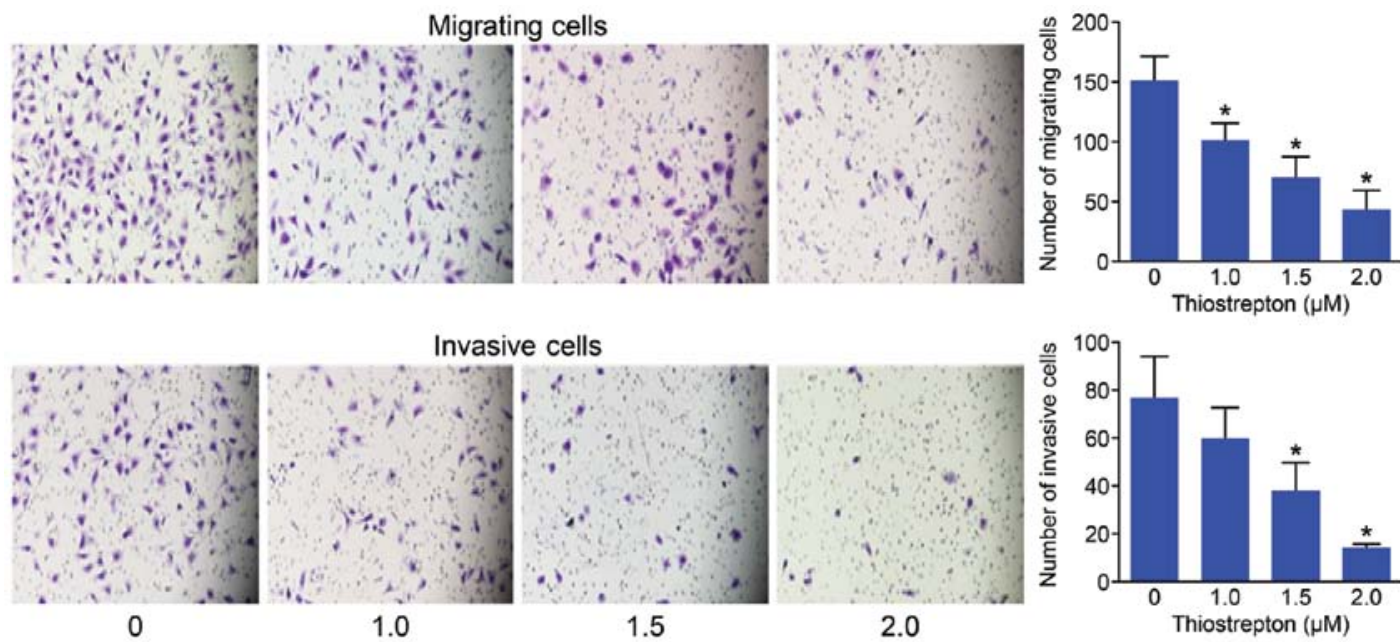

Invasive cells

Thiostrepton $(\mu \mathrm{M})$
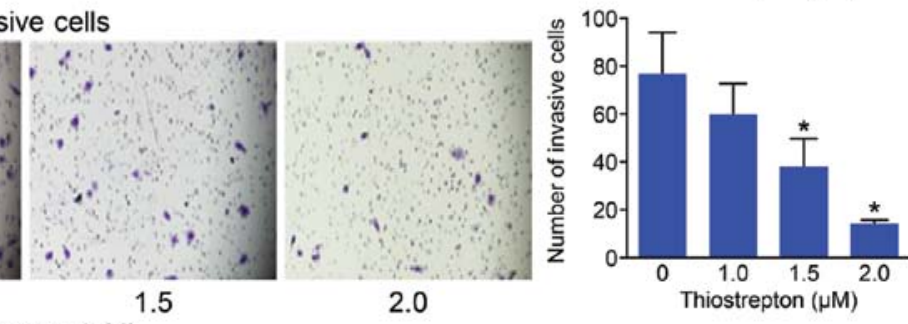

Figure 5. Thiostrepton inhibits the invasion and migration capacity of Daoy cells. Following treatment with the indicated concentrations of thiostrepton, the migration and invasion capacity of Daoy cells was measured by Transwell assay. ${ }^{*} \mathrm{P}<0.05$, statistically significant difference.

the single treatment group. Moreover, combination treatment resulted in greater increases in Bax, caspase-3 and PARP protein expression than either drug alone (Fig. 4B).

Thiostrepton impairs the migration and invasion of Daoy cells. FOXM1 play a critical role in tumor cell metastasis (27-29). Since tumor cell migration and invasion are essential steps in tumor metastasis, we further examined the effects of thiostrepton on cell migration and invasion in Daoy cells. Our data clearly showed that thiostrepton inhibited the migration and invasion capacity of Daoy cells in a dose-dependent manner with minimal involvement of cell inhibition (Fig. 5).

\section{Discussion}

As the most common malignant brain tumor in children, medulloblastoma is characterized by aggressive invasion and early metastasis. Current standard therapies have unsatisfactory effects on survival, and therapy-associated side-effects have led to a concentrated search for novel therapeutic approaches for MB. Here, we report for the first time that thiostrepton inhibits FOXM1 in Daoy MB cells. We found that thiostrepton inhibited Daoy cell proliferation and triggered apoptosis in a dose-dependent manner. We further demonstrated that thiostrepton chemosensitized Daoy cells to cisplatin by enhancing cisplatin-induced apoptosis. Thiostrepton also showed important antiprogressive effects such as inhibition of migration and invasion in Daoy cells.

We found that thiostrepton significantly inhibited the proliferation of Daoy cells. The $\mathrm{IC}_{50}$ value of thiostrepton in Daoy cells after $48 \mathrm{~h}$ of treatment was $1.69 \mu \mathrm{mol} / \mathrm{l}$, suggesting the sensitivity of Daoy cells to thiostrepton is comparable to that of other cancer cell types $(20,22)$. We next performed colony formation assays, which are an excellent indication of the long-term survival of tumor cells. These assays showed that thiostrepton strongly suppressed the ability of Daoy cells to form colonies. Notably, $1.75 \mu \mathrm{M}$ thiostrepton treatment had a more marked effect on colony formation than on cell proliferation (100 vs. $35.81 \%$ inhibition, respectively). These data suggest that thiostrepon may impact tumor cell self-renewal in addition to mitosis.

Furthermore, our studies demonstrated that thiostrepton treatment induced cell cycle arrest at the G2/M phase transition and triggered apoptosis. Similar results were obtained with RNAi-mediated inhibition of FOXM1 in Daoy cells. These results support the idea proposed by Priller et al (12) that FOXM1 inhibition results in failure of mitosis and leads to mitotic catastrophe in MB. Furthermore, our results demonstrated the efficient antitumor activity of thiostrepton in Daoy cells and suggest that the effects of thiostrepton may depend on the downregulation of FOXM1 target genes, which have been shown to be involved in the regulation of cell growth, apoptosis, cell cycle and progression (10).

Previous studies suggest that silencing of FOXM1 enhances sensitivity to chemotherapeutic agents in a wide range of human cancers $(15,16,25)$. This prompted us to investigate whether thiostrepton-mediated FOXM1 inhibition sensitizes Daoy cells to chemotherapy. In this study, the $\mathrm{IC}_{50}$ value of cisplatin in Daoy cells decreased from 5.622 to $3.116 \mu \mathrm{mol} / 1$ when it was combined with thiostrepton $(1 \mu \mathrm{mol} / \mathrm{l})$. Notably, all of the synergistic effects identified in this study occurred when thiostrepton was combined with suboptimal concentrations of chemotherapeutic drugs. Cisplatin is a standard chemotherapy agent used in MB treatment; however, its use results in drug-related morbidities including hearing loss and renal dysfunction. Therefore, a combination therapy approach using cisplatin and thiostrepton would not only improve the effect of cisplatin but also reduce the adverse side-effects of cisplatin by allowing it to be used at lower doses.

The ability to trigger tumor cell death is critical for successful treatment with chemotherapeutic agents. In the present study, flow cytometric analysis revealed a more marked increase in apoptosis in cells treated with a combination of thiostrepton and cisplatin than in those treated with either drug alone. To further explore the mechanism underlying the increased efficacy of combination treatment, we examined proteins known to regulate apoptosis. Bcl-2 is best known for its role in preventing apoptosis and conferring resistance to 
chemotherapeutic agents in various cell lines (30), whereas Bax accelerates cell death in response to certain apoptotic stimuli. Cells treated with both thiostrepton and cisplatin displayed a greater decrease in $\mathrm{Bcl}-2$ protein expression than cells treated only with cisplatin, and thiostrepton also enhanced cisplatininduced increases in Bax, PARP and caspase- 3 protein expression. These results suggest that thiostrepton sensitizes Daoy cells to cisplatin partly through enhanced apoptosis, possibly via a caspase-3-dependent pathway.

FOXM1 has been reported to play a central role in tumor metastasis (27-29), and few studies have explored the antitumor activity of thiostrepton. In our study, thiostrepton significantly decreased the migration and invasion capacity of Daoy cells. As these processes are both necessary steps for metastasis, we believe that thiostrepton may be a promising agent with which to antagonize the highly metastatic behavior of MB.

Collectively, our findings showed that thiostrepton induces apoptosis and significantly decreases the proliferation and metastatic potential of Daoy cells. Thiostrepton also acts synergistically with cisplatin by enhancing cisplatin-induced apoptosis. These results demonstrate that thiostrepton may have wide therapeutic and/or adjuvant applications for MB chemotherapy. Thiostrepton may be particularly useful in very young children with metastatic disease who currently must receive more aggressive chemotherapeutics.

\section{Acknowledgements}

This study was supported by The National Natural Science Foundation of China (81001119), The Grant Awarded to New Teachers from the Chinese Ministry of Education (20110171120112) and The Fundamental Research Funds for the Central Universities (11ykzd06).

\section{References}

1. von Hoff K, Hinkes B, Gerber NU, Deinlein F, Mittler U, Urban C, Benesch M, Warmuth-Metz M, Soerensen N, Zwiener I, Goette H, Schlegel PG, Pietsch T, Kortmann RD, Kuehl J and Rutkowski S: Long-term outcome and clinical prognostic factors in children with medulloblastoma treated in the prospective randomised multicentre trial HIT'91. Eur J Cancer 45: 1209-1217, 2009.

2. Massimino M, Giangaspero F, Garrè ML, Gandola L, Poggi G, Biassoni V, Gatta G and Rutkowski S: Childhood medulloblastoma. Crit Rev Oncol Hematol 79: 65-83, 2011.

3. Gilbertson RJ: Medulloblastoma: signalling a change in treatment. Lancet Oncol 5: 209-218, 2004.

4. Leary SE and Olson JM: The molecular classification of medulloblastoma: driving the next generation clinical trials. Curr Opin Pediatr 24: 33-39, 2012

5. Wierstra I and Alves J: FOXM1, a typical proliferation-associated transcription factor. Biol Chem 388: 1257-1274, 2007.

6. Laoukili J, Kooistra MR, Brás A, Kauw J, Kerkhoven RM, Morrison A, Clevers H and Medema RH: FoxM1 is required for execution of the mitotic programme and chromosome stability. Nat Cell Biol 7: 126-136, 2005.

7. Koo CY, Muir KW and Lam EW: FOXM1: from cancer initiation to progression and treatment. Biochim Biophys Acta 1819: 28-37, 2012.

8. Liu M, Dai B, Kang SH, Ban K, Huang FJ, Lang FF, Aldape KD, Xie TX, Pelloski CE, Xie K, Sawaya R and Huang S: FoxM1B is overexpressed in human glioblastomas and critically regulates the tumorigenicity of glioma cells. Cancer Res 66: 3593-3602, 2006.

9. Bektas N, Haaf At, Veeck J, Wild PJ, Lüscher-Firzlaff J, Hartmann A, Knüchel R and Dahl E: Tight correlation between expression of the Forkhead transcription factor FOXM1 and HER2 in human breast cancer. BMC Cancer 8: 42, 2008.
10. Ahmed M, Uddin S, Hussain AR, Alyan A, Jehan Z, Al-Dayel F, Al-Nuaim A, Al-Sobhi S, Amin T, Bavi P and Al-Kuraya KS: FoxM1 and its association with matrix metalloproteinase (MMP) signaling pathway in papillary thyroid carcinoma. J Clin Endocrinol Metab 97: E1-E13, 2012.

11. Xia JT, Wang H, Liang LJ, Peng BG, Wu ZF, Chen LZ, Xue L, $\mathrm{Li} \mathrm{Z}$ and $\mathrm{Li} \mathrm{W}$ : Overexpression of FOXM1 is associated with poor prognosis and clinicopathologic stage of pancreatic ductal adenocarcinoma. Pancreas 41: 629-635, 2012.

12. Priller M, Pöschl J, Abrão L, von Bueren AO, Cho YJ, Rutkowski S, Kretzschmar HA and Schüller U: Expression of FoxM1 is required for the proliferation of medulloblastoma cells and indicates worse survival of patients. Clin Cancer Res 17: 6791-6801, 2011.

13. Carr JR, Park HJ, Wang Z, Kiefer MM and Raychaudhuri P: FoxM1 mediates resistance to herceptin and paclitaxel. Cancer Res 70: 5054-5063, 2010.

14. Millour J, Constantinidou D, Stavropoulou AV, Wilson MS, Myatt SS, Kwok JM, Sivanandan K, Coombes RC, Medema RH, Hartman J, Lykkesfeldt AE and Lam EW: FOXM1 is a transcriptional target of ERalpha and has a critical role in breast cancer endocrine sensitivity and resistance. Oncogene 29: 2983-2995, 2010.

15. Kwok JM, Peck B, Monteiro LJ, Schwenen HD, Millour J, Coombes RC, Myatt SS and Lam EW: FOXM1 confers acquired cisplatin resistance in breast cancer cells. Mol Cancer Res 8: 24-34, 2010.

16. Halasi M and Gartel AL: Suppression of FOXM1 sensitizes human cancer cells to cell death induced by DNA-damage. PLoS One 7: e31761, 2010

17. Kwok JM, Myatt SS, Marson CM,Coombes RC, Constantinidou D and Lam EW: Thiostrepton selectively targets breast cancer cells through inhibition of forkhead box M1 expression. Mol Cancer Ther 7: 2022-2032, 2008.

18. Hegde NS, Sanders DA, Rodriguez R and Balasubramanian S: The transcription factor FOXM1 is a cellular target of the natural product thiostrepton. Nat Chem 3: 725-731, 2011.

19. Bhat UG, Halasi M and Gartel AL: FoxM1 is a general target for proteasome inhibitors. PLoS One 4: e6593, 2009.

20. Bhat UG, Halasi $M$ and Gartel AL: Thiazole antibiotics target FoxM1 and induce apoptosis in human cancer cells. PLoS One 4: e5592, 2009.

21. Halasi M, Schraufnagel DP and Gartel AL: Wild-type p53 protects normal cells against apoptosis induced by thiostrepton. Cell Cycle 8: 2850-2851, 2009.

22. Halasi M, Zhao H, Dahari H, Bhat UG, Gonzalez EB, Lyubimo AV, Tonetti DA and Gartel AL: Thiazole antibiotics against breast cancer. Cell Cycle 9: 1214-1217, 2010.

23. Wang M and Gartel AL: Micelle-encapsulated thiostrepton as an effective nanomedicine for inhibiting tumor growth and for suppressing FOXM1 in human xenografts. Mol Cancer Ther 10: 2287-2297, 2011.

24. Wang Y, Wen L, Zhao SH, Ai ZH, Guo JZ and Liu WC: FoxM1 expression is significantly associated with cisplatin-based chemotherapy resistance and poor prognosis in advanced non-small cell lung cancer patients. Lung Cancer 79: 173-179, 2013.

25. Zhang N, Wu X, Yang L, Xiao F, Zhang H, Zhou A, Huang Z and Huang S: FoxM1 inhibition sensitizes resistant glioblastoma cells to temozolomide by downregulating the expression of DNA-repair gene Rad51. Clin Cancer Res 18: 5961-5971, 2012.

26. Xu N, Zhang X, Wang X, Ge HY, Wang XY, Garfield D, Yang P, Song YL and Bai CX: FoxM1 mediated resistance to gefitinib in non-small-cell lung cancer cells. Acta Pharmacol Sin 33: 675-681, 2012.

27. Park HJ, Gusarova G, Wang Z, Carr JR, Li J, Kim KH, Qiu J, Park YD, Williamson PR, Hay N, Tyner AL, Lau LF, Costa RH and Raychaudhuri P: Deregulation of FoxM1b leads to tumour metastasis. EMBO Mol Med 3: 21-34, 2011.

28. Lok GT, Chan DW, Liu VW, Hui WW, Leung TH, Yao KM and Ngan HY: Aberrant activation of ERK/FOXM1 signaling cascade triggers the cell migration/invasion in ovarian cancer cells. PLoS One 6: e23790, 2011.

29. Huang C, Qiu Z, Wang L, Peng Z, Jia Z, Logsdon CD, Le X, Wei D, Huang $S$ and Xie K: A novel FoxM1-caveolin signaling pathway promotes pancreatic cancer invasion and metastasis. Cancer Res 72: 655-665, 2012.

30. Kim R, Emi M, Tanabe K and Toge T: Therapeutic potential of antisense Bcl-2 as a chemosensitizer for cancer therapy. Cancer 101: 2491-2502, 2004. 